\title{
USO DE SIMULADORES 3D COMO ESTRATEGIA TECNOPEDAGÓGICA PARA LA TRANSFERENCIA DE CONOCIMIENTO EN EL APRENDIZAJE DE LA ANATOMÍA ANIMAL
}

\section{USE OF 3D SIMULATORS AS A TECHNOPEDAGOGICAL STRATEGY FOR THE TRANSFER OF KNOWLEDGE IN THE LEARNING OF ANIMAL ANATOMY}

\author{
Danilo Bonilla Trujillo \\ Médico Veterinario Zotecnista, Esp. en Pedagogía, MsC en Ciencias \\ Pecuarias - Universidad Nacional Abierta y a Distancia \\ danilo.bonilla@unad.edu.co
}

\section{Viviana Vanadia Villamil Reyes}

Médico Veterinario, MsC en Salud animal - Universidad Nacional Abierta y A Distancia viviana.villamil@unad.edu.co

\section{John Fredy Montes Mora}

Ingeniero de Sistemas, Especialista en Informática y Telemática Universidad Nacional Abierta y A Distancia john.montes@unad.edu.co

\section{RESUMEN.}

Durante los últimos años, las tecnologías de la información y la comunicación han contribuido de manera positiva al proceso de enseñanza-aprendizaje logrando que los docentes puedan proponer estrategias didácticas diferentes a las usadas en los sistemas tradicionales, en la presente revisión se pretende dar una mirada desde temas de importancia en el aprendizaje teniendo en cuenta que existen estrategias didácticas como el uso de la tecnología y la pedagogía, el uso de simuladores en la educación como herramienta de enseñanza y el uso de éstos últimos en el aprendizaje de la anatomía enfocada a la anatomía animal. En esta revisión se recurre a varias fuentes documentales que propician un acercamiento al empleo de la tecnología 3D para optimizar la transferencia de conocimiento como parte de las maniobras 0

DOI: https://doi.org/10.22490/ECAPMA.3414 
estrategias tanto pedagógicas como tecnológicas que posibiliten el aprendizaje de la anatomía animal.

Palabras Clave: Transferencia; conocimiento; simulador; anatomía. ABSTRACT.

The new and emerging technologies as in the case of information and communication (ICT) in recent years have produced a high effect on education because they energize, give another vision and expectations to the Teaching-learning process, enabling teachers to proposing other didactic strategies different from those used in traditional systems, in the present review it is intended to look at issues of importance in learning such as the transfer of knowledge, as there are teaching strategies from the use of technology and pedagogy, the use of simulators in education as a teaching tool and the use of the latter in the learning of anatomy focused on animal anatomy. In this review, we resort to several documentary sources that favor an approach to the use of 3D technology to optimize the transfer of knowledge as part of maneuvers or strategies both pedagogical and technological that enable the learning of animal anatomy

Keywords: Transfer; knowledge; simulator; anatomy.

\section{INTRODUCCIÓN.}

El conocimiento y su transferencia ha estado pasando por muchos cambios en las últimas décadas y en parte se debe a la emergencia de las nuevas tecnologías que como se sabe avanzan día a día y transforman entornos permanentemente, así pues, ya no se concibe el conocimiento como proceso de transferencia sino como acción de transferido ya que las instituciones de enseñanza se han transformado de igual manera impactadas por las nuevas mediaciones y los nuevos medios que obligan a los profesores a diseñar estrategias didácticas y pedagógicas novedosas acordes a los tiempos. Una de estas estrategias contempla el uso de simuladores que desean recrear lo real llevándolo a un plano modificable por la digitalización y virtualización de contenidos, logrando que como en el caso de la anatomía animal se pueda acceder a elementos dinámicos en tercera dimensión casi como si se observara el animal en su estado material pero con el adicional de poder revisar estructuras que en formatos de imágenes o de digitalización en otras dimensiones no se pueden captar por el ojo del aprendiz en su total claridad logrando que al 
contrastar con las estructuras reales logre una interiorización y significación del conocimiento.

\section{TRANSFERENCIA DEL CONOCIMIENTO}

Actualmente se reconoce que las Universidades propenden por desarrollar procesos de producción y transferencia de conocimiento para dar cumplimiento a sus mandatos misionales de investigación, academia y proyección social que son los fundamentales. De acuerdo a Osorio (2009) es por ello que las Universidades deben obtener nuevos conceptos y nuevos ordenamientos en lo académico y organizacional para poder gestionar, proteger y transferir el conocimiento aplicado. Cabe entonces preguntarnos qué es la transferencia de conocimiento aplicándolo a resolver dificultades, transferir un conocimiento tomado como realizar una evolución y de expansión de un producto, de una tecnología o de una invención o teoría desde su origen hacia entornos o contextos tanto sociales como económicos diferentes (Becerra, 2004). Aunque es importante reconocer que también existen otros tipos de conocimiento transferidos como son el sobreentendido o supuesto y aquel que es evidente o categórico, es decir lo tácito y explícito que son aspectos del conocimiento diferentes a producir tecnologías, aparatos o productos (Albino, Garavelli y Gorgoglione, 2004). Para autores como MarínCasanova (2016), terminando el siglo XX el desarrollo de nuevas tecnologías como las de Información y comunicación (TIC) introdujeron grandes cambios en la forma de transferir conocimiento pues lo llevaron a ampliar y enriquecer los conceptos logrando convertirlos en acciones y propuestas para los miembros de algunas comunidades de conocimiento usando medios ciberespaciales. Por último y sin ahondar en muchas aristas que puede tener la transferencia de conocimiento, para ValenciaRodríguez (2013) se puede señalar que el éxito de la transferencia del conocimiento radica en el lenguaje usado ya que si los que lo emiten y quienes lo reciben tienen un uso de lenguaje común se podrá generar un conocimiento previo o posterior de manera compartida.

\section{Estrategias didácticas para el aprendizaje con uso de innovaciones y TIC.}

Si se toma en consideración que las TIC ha transformado la transferencia del conocimiento y que una de las circunstancias según Gómez y Oyola (2012), es el diseño de nuevos materiales didácticos de uso electrónico que facilitan el aprendizaje colaborativo logrando un proceso más activo, dinámico y rompiendo las barreras del aprendizaje a distancia esto genera 
un rol de mayor apropiación del aprendizaje por parte del estudiante. Esto esta reforzado por autores como de la Torre y Domínguez (2012) al afirmar que el papel de quien aprende se torna más activo y el profesor pierde el protagonismo, el educando tiene a disposición muchas herramientas de aprendizaje tales como elementos digitales o recursos que se pueden usar varias veces y que contenga todo lo que se requiere para el aprendizaje dentro del mismo recurso conformando así un Objeto de Aprendizaje con metas de alcance precisas logrando tener identificación propia, almacenamiento fácil, accesibilidad, reusabilidad y otras características que pueden ayudan a generar procesos de enseñanza-aprendizaje en un entorno virtual. Por otro lado, según Jaimez, Miranda, Vázquez y Vázquez (2017), las instituciones educativas diseñan e impulsan el aprendizaje en diversas plataformas virtuales donde existe variedad de recursos disponibles, formatos para comunicación y que, aunque llegan a ser arbitrarias, es facultad del docente dinamizarlas, motivar los participantes y elaborar estrategias didácticas que permitan un eficaz aprendizaje. En el mismo sentido Salinas (2004), expresa que si bien en la educación a distancia los conceptos de aprendizaje están ausentes al usar digitalización y virtualidad éstos pueden ser aplicados acorde a las necesidades logrando superar incluso las deficiencias del sistema tradicional de aprendizaje al alcanzar una combinación adecuada de componentes tecnológicos, pedagógicos y organizativos.

\section{Usos de simuladores como herramienta para potenciar el escenario formativo}

Los procesos académicos de apropiación del conocimiento y de aprendizaje significativo realizados a través de escenarios tecno pedagógicos como los simuladores, le permiten a un estudiante, usuario o cliente, el poder experimentar, evaluar y validar una aproximación de una situación real, por el uso de servicios y procesos que se dan en un contexto real, garantizando así experiencia muy cercana tal y como se haría en condiciones de un sistema vivencial, por ende la simulación puede brindar espacios seguros, de alta confiabilidad y riesgos bajos vitales de quien participa en dichos espacios formativos.

Con base en lo anterior, los simuladores son programas que permiten modelar la realidad, permitiendo que sus usuarios exploren progresiva o gradualmente con el escenario tecnológico, tener interacciones y realimentaciones automáticas que a partir de allí el estudiante pueda inferir y deducir, obteniendo un aprendizaje significativo. Esta realidad 
simulada, con características específicas acordes con las necesidades de formación: visual, sonora o aumentada hasta la posibilidad de incorporar tecnologías 3D, 4D y 5D pasando de un escenario bidimensional a escenarios aumentativos de transmedia, los cuales le garantizan al usuario el poder tener una vivencia muy cercana a una experiencia real que es percibida a través de los sentidos. La realidad 3D permite diseñar e implementar escenarios experimentales cercanas a la realidad, lo cual conduce a la idea, en términos "...de que los simuladores permiten repensar el contenido mismo, ubicándolo como una pequeña parte de todo el conocimiento posible a ser capturado, por lo cual, avanzar hacia las simulaciones finalmente significa entrar en una nueva era de historia y conciencia, llevando a nuevas formas de pensamiento y comprensión del mundo, y aceptando algunas limitaciones mayores en lo que se sabe y lo que se ha estudiado" (Aldrich, 2009).

La popularización del uso de simuladores en la educación tendrá un potencial transformador: Ver el mundo y representarlo a través de la aproximación de una simulación y no de un libro, requiere nuevas herramientas e incluso una nueva sintaxis con su correspondiente guía de estilo, pero creará una nueva generación de académicos y una nueva generación de líderes (Aldrich, 2009, p. 13).

El ecosistema digital ha cambiado, así como el productivo ha evolucionado, y por estar en la era de la Internet de las Cosas, es frecuente la prestación y adquisición de servicios especializados, la prioridad está más ligada con aspectos relacionados desde lo informativo, la experimental, conceptual, científico y tecnológico, en definitiva, la sociedad del conocimiento.

En la sociedad del conocimiento y de la economía naranja, la educación no puede ser indiferente a estos cambios tecnológicos y vertiginosos, la Universidad no puede seguir transfiriendo conocimientos utilizados los métodos planos o lineales como el de memorizar y recordar, sino direccionar el proceso de aprendizaje en aspectos como: discernir la información, capacidad crítica, inferir e innovar, formando estudiantes flexibles y adaptables que en la sociedad del conocimiento la enseñanza se "maneja" a sí misma, pues todo tiene que ver con el aprendizaje con su articulación mediada por las nuevas tecnologías de la información y comunicación.

Las dinámicas educativas han evolucionado en coherencia con los sectores: industrial, electrónico, telemático y computacional. Por ende, los procesos comunicacionales en doble vía donde el uso de medios 
educativos con multiformatos ligados con aprovechamiento de la era digital, exigen nuevos productos en multicontexto que estén satisfaciendo las necesidades formativas del estudiante de hoy: dinámico, interactivo y novedoso.

Cuando se establece comunicación entre el docente y el estudiante, no solo debe haber transmisión de información, lo cual se materializa en un aprendizaje corto que como consecuencia el aprendizaje será muy volátil, requiriendo procesos de repetición lineales no significativos. Es por ello, que el aprendizaje electrónico o conocido por sus siglas en inglés ( $E$ learning), cambia considerablemente la forma como se aprende, con un docente innovador, promotor de uso de nuevas herramientas tecnológicas, así como el desarrollo constante y a la medida de nuevos productos y objetos de gran riqueza académica rompiendo con el paradigma lineal y conservador. Este aprendizaje tradicional que aún se conserva cuando el docente no es innovador se traduce en procesos repetitivos y no contextualizados. Es aquí donde la innovación y la incorporación de nuevas tecnologías (NT) como la gamificación y simulación son insumos valiosos para la generación de nuevos objetos digitales académicos alienados con los nuevos estilos de aprendizaje.

Aprender no es complejo ni complicado, pero se requiere que tanto el docente como el estudiante estén siempre apoyados con herramientas tecnológicas adecuadas que permitan desarrollar competencias acordes con el propósito de formación. Dentro de este proceso de aprendizaje, es importante que el estudiante pueda experimentar, investigar, que aprenda del error, valide los riegos, de resolución a los problemas, cumpla los objetivos y adquiera un criterio más estructura a la hora de tomar decisiones.

Una estrategia innovadora y que permite este tipo de reforzamiento y de capacitación individual y colectiva son los simuladores y laboratorios virtuales. El usuario podrá experimentar, interactuar, repetir, ganar confianza y sobre todo familiarizarse con ambientes cercanos a la realidad, para aprender a manejar posibles situaciones y del cómo reaccionar ante éstas, siguiendo la lógica del aprendizaje y aumentando su respectiva curva del aprendizaje, el aprender-haciendo es una actividad que siempre estará presente en estos escenarios tecnológicos. Por ende, instituciones de educación superior necesitan herramientas de apoyo a los programas académicos para que los estudiantes trabajen, enriquezcan su proceso formativo y que aporten desde sus experiencias personales al mejoramiento de los contenidos digitales, garantizando de 
esta manera, una actualización progresiva del simulador y del laboratorio virtual.

El aprendizaje a través del uso de simuladores es uno de los métodos que ha demostrado ser muy eficaz para adquirir habilidades y destrezas. La mayoría de los contenidos de e-learning incorporar este tipo de escenarios formativos, por ende, el docente debe estar preparado y en continuo crecimiento de su curva de aprendizaje para que de esta manera la inserción de nuevos elementos multimediales no se convierta en una tarea tediosa, sino práctica y fácil de articular.

\section{¿Qué es un simulador?}

Es un software de computador que permite representar situaciones reales que son aplicables a cualquier saber o campo del conocimiento. El proceso de simulación pone a disposición del estudiante aspectos de usabilidad, aplicabilidad y experimentación con el fin de probarlas y evaluarlas para inferir y obtener un aprendizaje significativo. Se pueden encontrar en el mercado diversos simuladores destinados para las áreas como la(s): matemáticas, física, electrónica, matemáticas financieras, medicina, telecomunicaciones, softwares interactivos y educativo, entre otros. De las características que se pueden destacar de los simuladores, se tienen:

- Emular una situación real.

- $\quad$ Aprender del ensayo y error.

- $\quad$ Toma de decisiones a través de procesos inferenciales.

- $\quad$ Aumento de la curva de aprendizaje.

- $\quad$ Adaptaciones frente al cambio.

- $\quad$ Cambio de roles, acciones y cambios actitudinales.

\section{¿Qué es un laboratorio virtual?}

Un laboratorio virtual (LV) es un escenario tecnológico que permite el adiestramiento y desarrollo de pruebas que a través de la incorporación de experimentos controlados y no controlados acordes con condiciones básicas o muy cercanas al componente práctico o laboratorio real (LR), le permiten al estudiante desarrollar competencias que son reforzadas a través de los procesos repetitivos que permite el laboratorio virtual 
evitando el desgaste de insumos o elementos físicos necesarios para la práctica.

Las ventajas de los laboratorios virtuales que se pueden destacar son:

- Simulación de fenómenos cercanos a la experimentación real.

- $\quad$ Acceso a equipamiento avanzado usando el telecontrol sin tener ningún tipo de riesgo.

- $\quad$ Disminución de costos comparados con los laboratorios tradicionales.

- $\quad$ Autoaprendizaje progresivo, el estudiante puede ajustar elementos de entrada y configurar nuevos escenarios de aprendizaje acordes con sus niveles de exigencia y profundización de las temáticas.

- A través del ensayo y error, la experimentación es sin riesgo alguno.

- $\quad$ El límite de tiempo para el desarrollo de la práctica está bajo control del estudiante.

Es, por tanto, que los simuladores y/o laboratorios virtuales se convierten en el escenario ideal para afianzar los procesos de profundización y transferencia de conocimiento.

\section{Los simuladores en el aprendizaje de la anatomía.}

Para López y Visbal (2007) simular es un tipo de técnica que ha servido para formar y educar médicos desde hace más de cuatro décadas y que ha permitido a estudiantes y docentes, contar con una herramienta que perfeccione las técnicas de estudio y comprensión de la morfofisiología humana y animal. El diseño de un modelo a partir de un sistema real buscando comprender su funcionamiento y comportamiento, puede mejorar la perspectiva del aprendizaje tanto de docentes como estudiantes y se constituye en una excelente herramienta para correlacionar los conceptos teóricos con la práctica (Cohen, 1999).

En este contexto, a nivel mundial algunos investigadores e instituciones educativas concluyen que las Universidades deben adaptarse a las sociedades del conocimiento, destacando el rol que juegan las TIC dentro del desarrollo de estas acciones, convirtiéndose en un reto constante para la formación de pregrado y posgrado. Para el área de la salud, las TICs han permitido que los estudiantes y médicos estén actualizados permanentemente $y$ que puedan llevar a cabo experiencias diversas 
empleando entornos virtuales de aprendizaje en diferentes áreas como bioquímica, histología y anatomía, complementando el estudio teórico de estas temáticas. La mayoría de estos estudios arrojan resultados favorables de acuerdo a la aceptación y satisfacción por parte de los alumnos por la metodología empleada y la actualización de contenidos (Meléndez, 2009).

Los diferentes soportes visuales como bioimágenes, videos y contenidos multimedias, pueden ser considerados como una solución didáctica durante la presentación de las tareas de aprendizaje, lo cual se viene planteando desde hace varios años y permite modificar la dependencia absoluta de los materiales de origen cadavérico (Tamayo, 2017). En este nuevo escenario, entran didácticas de estudios basados en problemas, modelos plastinados y sintéticos, pinturas corporales, software, realidad virtual, entre otros. De esta forma, se ha empleado el uso de software con tecnología 3D que incluye un modelo de aprendizaje integral, en donde los estudiantes pueden interactuar de manera sencilla con los diferentes sistemas del cuerpo humano y que puede ser trabajado desde cualquier computador (Evans, 2008). En estos, los órganos 3D son reconstruidos a partir de imágenes obtenidas por tomografías, resonancias magnéticas y fotografías a partir de proyectos humanos como en el caso del "Visible Human Project" de la Biblioteca Nacional de Medicina de los Estados Unidos (Potes, 2018). En Colombia actualmente se aplica la anatomía tecnológica para la formación de los estudiantes de medicina humana. En diferentes dimensiones (3D) se logra un ambiente de interacción para observar interactivamente variadas estructuras anatómicas de los humanos, reduciendo los estudios en cadáveres que actualmente, no se están empleando en las Instituciones de educación superior (Turney, 2007). Pero estas herramientas tecnológicas no se están empleando únicamente en el estudio de la anatomía humana; también, los docentes de anatomía animal están buscando implementarlas en la comprensión y aprendizaje de los sistemas de las diferentes especies animales. Algunos softwares de anatomía equina como Equino Anatomedia, han diseñado módulos que incluyen cabeza, cuello, tórax, abdomen, pelvis, miembros anteriores y posteriores, desarrollados a partir de imágenes de alta calidad, diagramas, audio y video clips que resaltan los diferentes detalles anatómicos (El Sharaby, Alsafy y El-Gendy, 2015).

Hace algunos años, la Universidad de Georgia en Estados Unidos, desarrolló un software denominado "The Glass Horse" (Scherzer et al., 2010). Este permite la visualización virtual de los órganos digestivos en 
el equino y su comprensión a través del sistema gastrointestinal. En 2003, la Universidad de Murcia en España a su vez desarrolló un software acerca del sistema musculoesquelético del perro, basado en fotografías y disecciones. Estos softwares representan los esfuerzos por parte de los docentes de anatomía animal para que los estudiantes puedan conocer de forma directa las diferentes estructuras anatómicas de manera virtual, teniendo en cuenta las limitaciones para realizar disecciones en cadáveres que se presentan actualmente (Gutiérrez et al., 2017). Estos programas se pueden entonces, incluir como una herramienta interactiva de preparación y revisión de contenidos y estructuras dentro de la anatomía práctica. Usando el programa, los estudiantes primero pueden identificar las estructuras anatómicas relacionadas con las diferentes regiones topográficas a través de las imágenes, diagramas de disección y también los clips multimedia. Adicionalmente, los estudiantes pueden usar el programa como una herramienta útil para discutir los lineamientos o procedimientos anatómicos aplicados, y cómo reforzar los principios fundamentales y relacionarlos con el animal in vivo (Brenton et al., 2007).

La apariencia de modelos con alta calidad tridimensional, animaciones y datos volumétricos son muy llamativos para estudiantes y profesionales. Aprender anatomía requiere una comprensión conceptual de anatomía tridimensional y una manipulación práctica de herramientas y tejidos (EI Sharaby, Alsafy y El-Gendy, 2015). Con el crecimiento notable en el uso de programas multimedia y contenidos presentes en Internet, los estudiantes pueden desarrollar habilidades tecnológicas, mientras relacionan estructuras que les permitan comprender el estudio de la anatomía animal de una forma interactiva y práctica.

Así pues las posibilidades de transferir conocimiento o en otras estrategias pedagógicas generar conocimiento de manera constructivista es posible en la actualidad usando las tecnologías de la información y comunicación y específicamente herramientas como los simuladores que en el caso de la Anatomía animal y pensando en un aprendizaje que una lo teórico con lo práctico deben incluir modelos en 3D para relacionar mejor los conceptos y porque no ya estar usando realidad virtual, realidad aumentada u otras herramientas tecnológicas que apoyen el mejor desempeño de los estudiantes en las áreas de la anatomía animal.

\section{BIBLIOGRAFÍA.}

Albino, V., Garavelli, A.C. y Gorgoglione, M. (2004). Organization and technology in knowledge transfer. Benchmarking y International Journal,11(6), 584-600. 
Aldrich, C. (2009) The complete guide to simulations \& serious games. San Francisco: Pfeiffer. ISBN: 0470462736

Becerra, M. (2004). La transferencia de tecnología en Japón. Conceptos y enfoques. Ciencia. Ciencia UANL, 2 (1), 6-15

Brenton, H., Hernandez, J., Bello, J., Strutton, P., Purkayastha, S., Firth, T. and Darzi, A. (2007). Using multimedia and Web 3D to enhance anatomy teaching. Comput Educ, (49), pp.32-53.

Cohen, L. (1999). Medical simulation is wave of the future. Canadian Medical Association, 160(4), p.557.

de la Torre, L. M. y Domínguez, J. (2012). Las TIC en el proceso de enseñanza aprendizaje a través de los objetos de aprendizaje. Revista Cubana de Informática Médica 2012:4(1)91-100

El Sharaby, A., Alsafy, M. and El-Gendy, S. (2015). Equine Anatomedia:

Development, Integration and Evaluation of an E-Learning Resource in Applied Veterinary Anatomy. International Journal of Morphology, 33(4), pp.15771584.

Evans, C. (2008). The effectiveness of m-learning in the form of podcast revision lectures in higher education. Computers \& Education, 50, 491-498.

Gómez, B. I. y Oyola M. C. (2012). Estrategias didácticas basadas en el uso de TIC aplicadas en la asignatura de física en educación media. Escenarios -Vol. 10, No. 1, Enero-Junio de 2012, págs. 17-28

Gutiérrez, J., Gómez Jaramillo, M., Sudel, G. and Prater, M. (2017). Anatomical knowledge in veterinary medical students in Chile. Investigación en Educación Médica, 6(22), pp.70-74.

Jaimez, C. R., Miranda, K. S.,Vázquez, E. y Vázquez, F. (2017). Estrategias didácticas en educación superior basadas en el aprendizaje: innovación educativa y TIC. Universidad Autónoma Metropolitana, Unidad Cuajimalpa. pp 224. ISBN (electrónico): 978-607-28-0980-2

López, J. and Visbal, L. (2007). Simulación, herramienta para la educación médica. Salud Uninorte, (23(1), pp.79-95.

Marín-Casanova, J. (2016). La innovación epistémica reticular: De la transferencia del conocimiento al conocimiento transferido. Opción, 32 (80), 112-140. 
Martínez Aldanondo, J. (2002). Contenidos en E--learning: El rey sin corona. [citado abril, 2007] disponible en:

http://www.gestiondelconocimiento.com/leer.php?colaborador=javitomar\&id= 246

Meléndez, B. (2009). Entornos virtuales como apoyo al aprendizaje de la anatomía en medicina. Investigaciones Andina, 19 (11), pp.97-98.

Osorio M. E. (2009). Transferencia tecnológica. Idesia (Arica), 27(1), 5-6. doi: $10.4067 /$ S0718-34292009000100001

Potes, A. (2018). Icesi, única universidad en Colombia con Software de Anatomía interactiva en 3D. [online] Icesi.edu.co. Recuperado de https://www.icesi.edu.co/unicesi/todas-las-noticias/1201-icesi-unicauniversidad-en-colombia-con-software-de-anatomia-interactiva-en-3d.

Scherzer, J., Buchanan, M., Moore, J. and White, S. (2010). Teaching Veterinary Obstetrics Using Three-Dimensional Animation Technology. Journal of Veterinary Medical Education, 37(3), pp.299-303.

Tamayo, S. (2017). Aportes de los entornos virtuales (EVEA) en el proceso de enseñanza aprendizaje de la anatomía humana. Revista Argentina de Anatomía Clínica, (9 (1), pp.6-8.

Turney, B. W. (2007). Anatomy in a modern medical curriculum. Annals of Royal College of Surgeons of England, 89, 104-107.

Valencia-Rodríguez, M. (2013). Generación y transferencia de conocimiento. Ingeniería Industrial, 34(2), 178-187. Disponible en http://scielo.sld.cu/scielo.php?script=sci arttext\&pid=S181559362013000200007\&lng=es\&tlng=es 